\title{
Transcriptomics analysis and co-expression network revealing candidate genes for laccase activity of Lenzites gibbosa
}

Jie Chen

Northeast Forestry University

Yujie Chi (D 18704633148@163.com )

Qingquan Zhao

Northeast Forestry University

Xin Hao

Northeast Forestry University

Research article

Keywords: White-rot fungus, laccase, WGCNA, TCA, mitochondria

Posted Date: May 29th, 2020

DOI: https://doi.org/10.21203/rs.3.rs-31352/v1

License: (c) (1) This work is licensed under a Creative Commons Attribution 4.0 International License.

Read Full License 


\section{Abstract \\ Background}

Lenzites gibbosa is a common white-rot fungus of Polyporaceae in the cold temperate zone which cause spongy white decay of wood.

\section{Results}

In this study, the lignin degradation pathway of L. gibbosa at 5-time points under wood treatment was studied by RNA-Req technology. A total of 5232 DEGs were identified from 15 libraries. In 0-3 days, the mycelia are in the adaptive stage, and the mycelia began to proliferate within 3-5 days. After 5 days, the number of DGEs decreased significantly, the mycelium growth entered the platform stage, and the life activity was basically stable. In the secondary metabolism, oxidoreductase such as laccase, 2oxoglutarate-Fe (II) type oxidoreductase, peroxisomal hydratase-dehydrogenase, dual-functional monooxygenase dominated and increased steadily, and manganese peroxidase appeared in the middle stage. With the accumulation of lignin intermediate products, P450 and ABC transporters were from the inhibition to the activation. Weighted gene co-expression network analysis (WGCNA) showed that among the 24 modules 6 modules were significantly correlated with laccase activity and the most correlated were turquoise and blue module. The central hub genes were also identified, including gene_7458, gene_61, gene_7458, gene_1741, gene_11087 which were consistent with the DGEs. These genes have high connectivity, module membership, and gene significance in the module. The enrichment analysis of GO and KEGG pathway indicated that the genes involved in cell cycle, citrate cycle (TCA cycle), nicotinate and nicotinamide metabolism, mitochondrial outer membrane, succinate dehydrogenase, carboxypeptidase and exopeptidase activity, flavin adenine dinucleotide binding, oxidoreductase activity, acting on the $\mathrm{CH}-\mathrm{CH}$ group of donors, quinone or related compound as acceptor pathway were highly related to laccase synthesis pathway. Construction of gene co-expression network and hypothetical $L$. gibbosa laccase synthesis pathway.

\section{Conclusion}

This study focused on the screening of $L$. gibbosa degradation of lignin-related genes. And, this is the first study reporting co-expression patterns of a gene network in L. gibbosa laccase activity after wood treatment which is helpful to understand the synthesis pathway of laccase and improve the activity of laccase, which can be used to increase the rate of lignin degradation by L. gibbosa in the further.

\section{Background}

White-rot fungus have been widely studied as one of the most effective microorganisms for lignin degradation. White-rot fungus can decompose lignocellulose and remove toxic compounds by secreting 
lignin-decomposing enzymes, such as manganese peroxidase, lignin peroxidase, multifunctional peroxidase and laccase [1,2]. Laccase is a multicopper oxidoreductases containing four copper ions, which exists in plants, fungi, insects, and bacteria [3-6]. In fungi, laccase is studied to have many physiological functions, including defense [7], melanin synthesis and lignin degradation [8]. In recent years, it has been found that laccase has high application value which can be used in paper bleaching, even a biological fertilizer and the degradation of refractory pollutants [9-11]. So far, most laccase research was about laccase genes cloned. For example, laccase genes have been cloned from Phanerochaete chrysosporium [12], Fome lignosusand [13], Pycnoporus cinnabarinus [14] and Trametes sp strains [15]. However, a single clone of laccase is not enough, the comprehensive metabolic system needs to be explored. This is helpful to understand the synthetic pathway of laccase in cells and improve the activity of laccase.

Lenzites gibbosa is the one of white-rot fungus that is widely distributed in the cold temperate zone. With a fast growth rate and a strong ability to decompose wood, L. gibbosa can decompose lignin by enzymatic system and enzymatic properties. In our previous study, we have been found that the activity of manganese peroxidase, lignin peroxidase, laccase on the sawdust matrix of poplar $[16,17]$. Two fulllength genes of laccase isoenzyme cDNA, and three MnPs genes [19] have been cloned from this species. There are still many redox enzymes in the lignin decomposition enzyme system that have not been studied. However, it is still unknown if these enzymes are essential for lignin decomposition. Weighted correlation network analysis ((WGCNA)) is an R software package that correlation the method for describing and visualizing data point networks [20]. It can find related pathways and genes that play an important role in transcriptional regulation, and can easily pre-predict unknown gene regulatory relationships through known genes in the network. It is widely used in humans, animals and plants, but it is rarely used in fungus [21-23].

In this study, 5-time points for transcriptome analysis for the wood treatment. The purpose of this experiment was to study the effect of wood on the secondary metabolism of L. gibbosa during growth. Therefore, a large number of differentially expressed genes (Deg) were obtained. Then, laccase activity was used as a trait, and WGCNA was used to construct a co-expression module using expression data. The enrichment analysis of KEGG pathway in DEGs was carried out on the modules with biological significance, and the key hub genes in each module were identified, with highly linked in each meaningful KEGG pathway. The current research needs to specifically focus on the genomics-based approaches to optimization of the fungal lignin degradation system strengthen the extracellular enzyme system, and comprehensive metabolic structure design is needed, this is the first study reporting WGCNA of a gene network in white-rot fungus which is helpful to understand the synthesis pathway of laccase and improve the activity of laccase, which can be used to increase the rate of lignin degradation by L. gibbosa in the further. This meets the various needs of human beings in clean bioenergy, industrial uses, and environmental protection.

\section{Results}




\section{Overall transcriptome and sequencing data}

Total RNA extraction and RNA-Seq analysis were carried out on the mycelia of 0 days, 3 days, 5 days, 7 days, and 11 days after wood chip treatment, and the cleaning data of $114.55 \mathrm{~Gb}$ were obtained. A total of 15 libraries were constructed, named CK 1-3, MX1 1-3, MX2 1-3, MX3 1-3, and MX4 1-3. The reading produced by Illumina sequencing had a length of $150 \mathrm{BP}$, and the percentage Q score of Q30 bases was not less than $91.84 \%$ (Table S1). The reference genome of this species is Tragib1 in JGI (http://genome.jgi.doe.gov/Tragib1/Tragib1.home.html). The comparison efficiency of sample data of all 15 transcriptional groups was between $88.51 \%$ and $91.37 \%$, which belonged to a high level and met the needs of follow-up analysis.

\section{Identification of DEGs and functional enrichment analysis of the genes}

By analyzing the transcriptome data of L. gibbosa at different time points of wood chip degradation, a total of 5232 DEGs were identified. Among them, 1803 genes were significantly up-regulated and 3429 genes were significantly down-regulated (Table S2).

We carried out GO classify analysis of DEGs. The genes were distributed in 38 categories of biological process, cell composition, and molecular function (Table S4). In the different groups, the DEGs in biological processes were mainly related to cellular process, metabolic process, single-organism process, biological regulation, and response to the stimulus. Catalytic activity was $\mathrm{GO}$ terms the most significantly in molecular function.

We carried out the GO enrichment analysis of adjacent time point DEGs. The GO with significant difference among the four time periods was 221,135,132,132 (Fig. 1). Under the stimulation of wood chips, the number of significant differences in go was more in 0-3 (CKvsMX1) days $(p<0.05)$, and then tended to be stable with time. At the same time, a total of 49 go are enriched in 4 subsets, of which it is worth noting that transition metal ion transport (G0:0000041), phenylpropanoid catabolic process (GO:0046271), lignin catabolic process (GO:0046274), lignin metabolic process (G0:0009808), flavin adenine dinucleotide binding (GO:0050660), antioxidant activity (GO:0016209), oxidoreductase activity, acting on the aldehyde or oxo group of donors, NAD or NADP as acceptor (G0:0016620)ख82 G0 were significantly enriched in 3 subsets. Alpha-amino acid catabolic process (GO:1901606), oxidoreductase activity, acting on a sulfur group of donors, disulfide as acceptor (G0:0016667) were significant difference only in 3-5 days (MX1vsMX2). And, intracellular (G0:0005622) was especially in 5-7 days.

To evaluate the biological and functional significance of DEGs, pathway enrichment analysis was carried out using the KEGG database. Among the 10 groups, a total of $403378,373,462,8,26,25,3$, 9, and 5 genes were annotated in the KEGG database. The change of gene expression of the strain was different at different times after wood chips stimulation, which changed greatly in 0-3 days and little in the later stage (Fig. 2). Significant pathways $(p<0.05)$ were mainly related to Amino sugar and nucleotide sugar metabolism pathway (ko00520), Biosynthesis of antibiotics pathway (ko01130), Butanoate metabolism 
pathway (ko00650), Carbon metabolism pathway (ko01200), Cell cycle - yeast pathway (ko04111), Citrate cycle (TCA cycle) pathway (ko00020), Glycolysis / Gluconeogenesis pathway (ko04113), Meiosis - yeast pathway (ko00010), Alanine, aspartate and glutamate metabolism (ko00250) (Table S3). There were 29 pathways only enriched in CK groups (CK vs MX1, CK vs MX2, CK vs MX3, and CK vs MX4), caused by wood chips stimulation (Table S3). And Valine, leucine and isoleucine biosynthesis (ko00290), Biosynthesis of antibiotics (ko01130), Ascorbate and aldarate metabolism (ko00053), and Tyrosine metabolism (ko00350) were same as other groups. Carbon metabolism (ko01200), Butanoate metabolism (ko00650), Glycolysis / Gluconeogenesis (ko00010), Biosynthesis of antibiotics (ko01130), Taurine and hypotaurine metabolism (ko00430), Galactose metabolism (ko00052), Citrate cycle (TCA cycle) (ko00020), ABC transporters (ko02010) were special significantly enriched ( $p<0.05$; Table S3).

4 DEGs were selected randomly for qRT-PCR validation. Levels of log2FC associated with these genes were indeed consistent by both RT-PCR and qRT-PCR (Fig. S1). Thus, qRT-PCR analysis confirmed that the changes detected in the RNA sequencing analysis were reliable.

\section{Construction and analysis of the weighted gene co- expression network}

Finally, 12329 genes were selected for WGCNA. When $\beta=22$, the fitting index of scale-free network $R^{2}=$ $0.85>0.8$, and the average connectivity approached 0 , indicating that the scale-free network can be obtained by power processing with this value. So $\beta=22$ was chosen to construct the scale-free network (Fig. 3). The dynamic clipping orithm was used to cluster and divide the genes into modules, and the module feature vector (min Module Size $=30$ ) of each module is calculated. By clustering the ME distance, the clustering of the module is constructed. Modules with high K.in were at the top of the branch because they showed the highest interconnectivity with the rest of the module. The horizontal line represented the threshold (CutHeight $=0.2$ ) used to define the meta-module (Fig. S2). With the combination of similar modules, a total of 24 gene co-expression modules were obtained, each of which was marked with different colors and varies were from 45 to 3154 genes (Fig. 4). In order to further quantify the co-expression similarity of the whole module, we got the correlation of 24 modules based on heat map and clustering according to the expression of genes. Each module also showed independent verification of each other, with gradually saturated blue and red indicating high co-expressed interconnectedness (Fig. S3). There was a negative correlation between the blue and turquoise module and a positive correlation between the tan module.

A. Module division by dynamic tree cut, different colors represent different modules; B. Module division after merging similar modules

\section{Identification of meta-modules related to the laccase activity}

As we know, ME is the first principal component of a given module and can be regarded as the representative of the gene expression profile of the module. To identify modules significantly related to 
laccase activity, the relationship between each module and laccase activity was evaluated based on the correlation between ME and laccase activity (Fig. 5B). The change of laccase activity included 15 samples, and the correlation map between sample and laccase activity was constructed (Fig. 5A). The results showed that among the 24 modules, 6 modules were highly specific to laccase activity $(|r|>0.60$ and $p<0.001$ ), including turquoise, lightpink4, tan, pink, grey, blue. Among them, laccase activity was a significant negative correlation to turquoise module $(r=-0.856128099, p=5 e-05)$ and positive correlation to blue module $(r=0.944398154, p=1 e-07)$.

The genetic composition of specific modules that most closely related to laccase activity, we studied the network-specific properties of GS and MM for calculating the ME value of a single module and genes. If the content of GS and MM of a gene was higher, then its characteristic was more meaningful [24]. Therefore, for a particular module, the higher the $r$-value and the smaller the $p$-value were which proved that the module members were more representative of the module characteristics. Blue module (cor = $0.82, p<1 e-200$ ) (Fig. 5C), turquoise module (cor $=0.81, p=1.6 e-172)$ (Fig. 5D) and $\tan (\operatorname{cor}=0.59, p=$ 9.4e-135) (Fig. 5E) module may play a more important biological role in the process of laccase activity.

\section{Identification of laccase activity hub genes and network construction}

The 1329 hub genes were selected $\mathrm{GS}>0.8, \mathrm{P}<0.01$ and $\mid \mathrm{MM}$.blue $\mid>0.8$. To explore the interesting genes of blue module hub genes, these interesting genes usually have higher GS, as well as higher MM and K.in. Therefore, based on the above three indicators, the top interesting genes (Top30) in the blue module were listed in the Venn map, and five genes were crossing points (Fig. 6A and TableS5). Similarly, Cytoscape (3.7.1) was used to model the network view of the five interesting genes of the blue module (threshold $=$ 0.02) (Fig. S4 and TableS6). The value range of each gene in the blue module network view based on these three parameters was as follows: the K.in count range (4.72 183.70, the average 63.80); GS score range ( $0.80 \sim 0.98$, the average was 0.86$)$; $\mathrm{MM}$, count range $(-0.96 \sim 0.99$, and the average was 0.78$)$. Also, 66 genes were highly linked to central genes by the Cytoscape construction network(Fig. 6B). Specifically, 4 transcription factors were predicted, including gene_9051, gene_9414, gene_9848, gene_9806 in 66 highly relevant genes (Fig. 6B) $\square$

The 160 hub genes were selected $G S>0.9(P<0.01$ and $\mid M M$. turquoise| $>0.8)$. Among the core genes of the turquoise module, the top interesting genes (Top50) of the high GS, higher MM, and K.in were listed in the Venn map, and seven genes were crosses (Fig. 7A and TableS6). Similarly, Cytoscape was used to model the network view of the seven central genes of the turquoise module (threshold $=0.02$ ) (Fig. 7B and Tables7). The value range of each gene in turquoise module network view based on these three parameters was as follows: K.in counting range (12.57 169.98, the average 72.48); GS score range (0.90 0.98, the average 0.92); MM counting range $(-0.96 \sim 0.97$, the average -0.77$)$. Besides, 7 genes were highly linked to central genes by the Cytoscape construction network map (Fig. 7B and Table S18).

\section{Functional analysis of the two modules}


To better understand the function of these module genes in laccase secretion, we enriched the hub genes of blue and turquoise modules by GO terms, COG classification, and KEGG pathway classification and enrichment. In the module blue, a total of 444 hub genes were annotated for COG classification, and the class with most of them annotations were Q: Secondary metabolites biosynthesis, transport and catabolism, H: Coenzyme transport and metabolism, E: Amino acid transport and metabolism, T: Signal transduction mechanisms, G: Carbohydrate transport and metabolism, O: Posttranslational modification, protein turnover, chaperones (Fig. 8C). 725 hub genes have been annotated and enriched to $73 \mathrm{GO}$ terms (belonging to biological process, cellular component, molecular function, $\mathrm{P}<0.05$ ), most of them were enriched with protein refolding, ER-associated ubiquitin-dependent protein catabolic process, cell cycle process, sterol metabolic process, and these processes can be classified as biological processes. Most of them were enriched with nuclear part, mitochondrial outer membrane, site of double-strand break can be classified as cellular components. Many terms were also enriched including RNA binding, ATP binding, succinate dehydrogenase activity as molecular functions(Table S9 and Fig. 8B), 554 hub genes were annotated and enriched by KEGG, which mainly belong to cell cycle - yeast, meiosis - yeast, citrate cycle (TCA cycle), non-homologous end-joining, DNA replication, protein processing in endoplasmic reticulum, nicotinate and nicotinamide metabolism (ko00760) (TableS10,11, Fig. 8A, D). And, 74 transcription factors in 44 transcription factor families were predicted in the blue module (Table S12).

In the module turquoise, a total of 57 hub gene were annotated for COG classification, and the class with most of them annotations were C: Energy production and conversion, T: Signal transduction mechanisms, E: Amino acid transport and metabolism, Q: Secondary metabolites biosynthesis, transport and catabolism (Fig. 9B). 93 hub genes were annotated and enriched to $47 \mathrm{GO}$ terms (belonging to biological process, cellular component, molecular function, $\mathrm{P}<0.05$ ), most of them were enriched with protein targeting, establishment of protein localization to organelle, proteolysis involved in cellular protein catabolic process, modification-dependent protein catabolic process, mitochondrion localization, ubiquitin-dependent protein catabolic process can be classified as biological processes. Most of them were enriched with mitochondrial outer membrane, SAGA-type complex, cytoskeletal part can be classified as cellular components. Many terms were also enriched including RNA binding, succinate dehydrogenase activity, oxidoreductase activity, acting on the aldehyde or oxo group of donors, NAD or NADP as acceptor, oxidoreductase activity, acting on the $\mathrm{CH}-\mathrm{CH}$ group of donors, quinone or related compound as acceptor can be enriched as molecular functions (Table S13, Fig. 9C). 72 hub genes were annotated and enriched by KEGG, which mainly belong to cellular processes, genetic information processing, metabolism pathway. More specifically, there were mainly cell cycle - yeast, citrate cycle (TCA cycle), meiosis - yeast, oxidative phosphorylation, nicotinate and nicotinamide metabolism, basal transcription factors, carbon metabolism (TableS14,15, Fig. 9A, D). And, seven transcription factors in seven transcription factor families were predicted in the turquoise module (gene_10591, gene_7921, gene_4145, gene_40, gene_11087, gene_10898, gene_6727) (Table S16).

\section{Discussion}


We used RNA-Seq and WGCNA of gene-trait correlations to analyze L. gibbosa gene expression at 5 time periods. Our results indicated that the key hub genes related to laccase in wood treatment may play important roles in the identification of candidate biomarkers, and in understanding the molecular mechanism of laccase.

\section{Differential gene expression as a function of wood stimulus}

Time-series gene expression data provide trajectories of gene expression levels over time, so we can observe the continuity of all events during a time course through the identified differentially expressed genes (DEGs) at multiple time points along the time course, and then studying the enrichment of biological processes. In adjacent periods, transition metal ion transport (GO:0000041), phenylpropanoid catabolic process (GO:0046271), lignin catabolic process (GO:0046274), lignin metabolic process (GO:0009808), flavin adenine dinucleotide binding (G0:0050660), antioxidant activity (G0:0016209), oxidoreductase activity, acting on the aldehyde or oxo group of donors, NAD or NADP as acceptor (G0:0016620) were common enrichment. It is well known that white-rot fungus secrete extracellular enzymes (mainly laccase, manganese peroxidase, lignin peroxidase) to degrade lignin. These enzymes all need the transport of metal ions, $\mathrm{Cu}, \mathrm{Zn}, \mathrm{Fe}, \mathrm{Mn}[25,26]$. Also, coenzymes are a kind of compounds with special chemical structure and function, which widely participate in enzyme-catalyzed redox reactions and undertake the function of transferring electrons, atoms or groups in enzyme-catalyzed reactions, for example, NAD + or NADP + carry reducing hydrogen. Redox reaction is the main reaction process of lignin degradation, indicating that wood chip degradation has been going on. On the other hand, on the third day, the number of DGEs decreased significantly, indicating the early adaptation of the fungus to wood chips. At this time point, CytochromeP450, Glutathione S-Transferases (GSTs), and ABC transporters were significantly down-regulated, indicating that compounds released from biomass or newly produced in the process of wood chip degradation had active detoxification $[27,28]$.

At the same time, one down-regulated Laccase-3 and one up-regulated Laccase-1,4 up-regulated and 6 down-regulated in the different families of Glycosyl hydrolases were found in DEGs. The results showed that the laccase began to produce and the sugar group began to decompose. Citrate cycle (TCA cycle) (ko00020) including 6 down-regulated genes, Galactose metabolism (ko00052) including 5 downregulated genes, Glycolysis/Gluconeogenesis (ko00010) including 10 down-regulated genes significantly enriched $(p<0.05)$ indicated that TCA cycle, Galactose metabolism, Glycolysis/Gluconeogenesis was inhibited, and the expression of pyruvate kinase was also inhibited. More specifically, Carbon metabolism (ko01200) contained 43 differential genes down-regulated and 1 differential gene up-regulated. The lignin supplied by wood is phenol cyclic compound, Phenol compounds can be degraded through the Tyrosine Metabolism pathway (ko00350) to produces simple sugar.3 FAD-binding domain was up-regulated. Flavin adenine dinucleotide (FAD) binding domain activates peroxidase by producing $\mathrm{H}_{2} \mathrm{O}_{2}$ [29], and promotes lignocellulose decomposition by regenerating the LPMO active on cellulose through the REDOX cycle of phenols and corresponding quinone media [30]. It is well-known fungi growth requires a carbon source. In the early adaptation stage, the demand for carbon sources is relatively small. However, the inhibition of carbon metabolism and the TCA cycle were indicated that the metabolism of energy mainly 
depended on some other oxidoreductase such as Laccase, tyrosine kinase, Glycosyl hydrolase, and other oxidoreductases to decompose extracellular proteins and wood chips to provide energy. Stimulation of exogenous benzoic acid to white-rot fungus Phanerochaete chrysosporium, the levels of most TCA cycle organic acids, citrate, cis-aconitate, 2-oxoglurtarate, fumarate, and malate, dramatically and coordinately decreased. The accumulation of most amino acids and some fatty acids may be related to the decrease of the level of TCA intermediates[31], the same as our results. The expression of aspartyl protease, glutamine amidotransferase, and serine/threonine kinase related genes were up-regulated. Aspartic acid is the raw material for the indirect synthesis of isoleucine, threonine, methionine, lysine, and other amino acids, and amidotransferase is one of the key enzymes in the indirect synthesis of Glutamyl-tRNA [32]. Min.et al.,2002). The main function of aspartic protease is to degrade protein antigen and promote the activity of other enzymes (Li,2016), suggesting that the basic synthesis pathway of amino acids is downregulated, while the synthesis of amino acids through the indirect pathway is up-regulated, which is beneficial for the strain to better adapt to the change of nutritional conditions (Table S3, Table S17). Serine /threonine protein kinase (S/TK) is involved in protein phosphorylation [33]. Trichoderma reesei and Neurospora crassa have been shown to catalyze serine and threonine phosphorylation, thereby transferring the signal from extracellular to intracellular, and then promoting or inhibiting cellulase secretion by affecting downstream gene transcription or protein modification[34-36]. The mycelium began to proliferate in 3-5 days and was in the exponential stage. After 5 days, the number of DGEs decreased obviously, the mycelium growth entered the platform stage, and the life activity was basically stable.

Lignin degradation is closely related to secondary metabolism. Therefore, we analyzed the secondary metabolism to obtain detailed information about the metabolic disorder after wood chip treatment. At 011d, Laccase-1, 2-oxoglutarate-Fe(II) type oxidoreductase, Peroxisomal hydratase-dehydrogenase, Dualfunctional monooxygenase up-regulated steadily, the content of laccase increased steadily, and degraded lignin [37]. Intermediate period, NADPH-dependent 1-acyldihydroxyacetone phosphate reductase, Dualfunctional monooxygenase/methyltransferase psoF, Ferric reductase transmembrane component 1 , Manganese peroxidase 3 began to up-regulate and secrete, participate in lignin degradation. Especially at CKvsMX4, 4 P450 and 1 ABC transporter were up-regulated (Table S18). The degradation of lignin will produce by-products, which need fungal metabolism or detoxification. Studies have shown that the secretion of lignin active enzymes is related to the up-regulation of signal transduction (kinase) or detoxification related genes (cytochrome P450s, GSTs and ABC transporter). These genes play a role in the adaptation of fungi to lignin decomposition [38]. P450 mediated pentachlorophenol oxidation [39], the CYP512, CYP5136 and CYP5150 families can oxidize a wide range of hydrocarbons, including phytochemicals and key metabolic intermediates in fungi, such as steroids, indicating that they play a role in the primary and secondary metabolism of fungi [40]. Matsuzaki et al studied the intracellular changes of $P$. chrysosporium during benzoic acid metabolism at the proteome and metabolic level, and found that aryl alcohol dehydrogenase, aromatic aldehyde dehydrogenase and cytochrome P450 play a key role in benzoic acid metabolism[31]. As lignin conditions, the expression of microsomal protein of $P$. chrysosporium strain RP-78, which found that transport proteins and cytochrome P450s were involved in 
the degradation of lignin and played an important role [41]. In the secondary metabolism, lignin degradation was dominated by oxidoreductases such as laccase and increased steadily, P450 and ABC transporters were inhibited at first, and manganese peroxidase began to appear with the increase of fermentation time. At the same time, P450 and ABC transporters were activated by the accumulation of lignin intermediates. Under wood treatment, how the L. gibbosa coordinates with each other to degrade lignin, what secondary metabolic pathways do our results suggest play a key role.

\section{WGCNA}

Although many studies have studied gene expression about laccase, little attention has been paid to the analysis of laccase activity of complex co-expression networks. Here, we report the results of a WGCNA of wood treatment; 24 co-expression network modules were obtained. The modules turquoise, lightpink4, tan, pink, grey, and blue were significantly correlated with laccase in L. gibbosa $(|r|>0.60$ and $p<0.001)$. Also, the conservatism of the two modules in different data sets in- deeply studied. Based on the WGCNA meta-module and further analysis of network characteristics (GS, MM, and K.in), we confirm that blue and turquoise modules were the main modules. In GO classification, GO entries were highly consistent, mainly concentrated in the biological process: metabolic process; cellular process: cellular component, cell part, cell; molecular function: binding, catalytic activity; which were consistent with the pathway of laccase production in cells. In enrichment ( $p<0.05)$, we found that cellular component organization(G0:0016043), mitochondrial outer membrane(GO:0005741), RNA binding (G0:0003723), succinate dehydrogenase activity (G0:0000104), succinate dehydrogenase (ubiquinone) activity (G0:0008177), carboxypeptidase activity (G0:0004180), exopeptidase activity (GO:0008238), flavin adenine dinucleotide binding (GO:0050660), oxidoreductase activity, acting on the $\mathrm{CH}-\mathrm{CH}$ group of donors, quinone or related compound as acceptor (G0:0016635), translation factor activity, nucleic acid binding (G0:0008135) existed in two modules (Table S9, S13), The results showed that they were related to the secretion of laccase or co-expressed in the mycelium and carried out life activities together, which showed that they were related to the secretion or co-expressed of laccase and carried out life activities together. The activity of mitochondria was very active. Succinate dehydrogenase, the binding enzyme of mitochondrial inner membrane, belongs to cytochrome oxidase, which is one of the hubs connecting oxidative phosphorylation and electron transport. It is the only multi-subunit enzyme integrated on the membrane in the TCA cycle. At the same time, its auxiliary group is Flavin adenine dinucleotide (FAD), which binds to the histidine of enzyme protein by the covalent bond. In laccase oxidation, electrons are obtained from phenolic substrate molecules to form semiquinone radicals, and other oxidoreductases act on $\mathrm{CH}-\mathrm{CH}$ group instruments for the degradation of phenols. Exopeptidase and carboxypeptidase are proteolytic enzymes, catalyze the hydrolysis of the peptide bond at the end of the polypeptide chain and free the terminal amino acid. At the same time, the KEGG pathways of blue and turquoise modules were also highly consistent. In particular, the path analysis of these modules showed that Cell cycle yeast(ko04111), Citrate cycle (TCA cycle) (ko00020), Meiosis - yeast (ko04113), Nicotinate and nicotinamide metabolism(k000760) were the highest core pathway of blue and turquoise modules in the secretion of laccase. (Table S10,S11,S14,S15). Oxidoreductase activity, acting on the CH-NH2 group of 
donors, NAD or NADP as acceptor (GO:0016639), glutamate dehydrogenase (NAD+) activity (G0:0004352) existed only in the blue module. Oxidoreductase activity, acting on the aldehyde or oxo group of donors (G0:0016903) existed only in the turquoise module. We found that Nicotinate and nicotinamide metabolism was closely related to laccase activity. However, few studies have reported whether Nicotinate and nicotinamide metabolism will affect laccase activity. Therefore, whether Nicotinate and nicotinamide metabolism is related to laccase activity remains to be further verified.

After extensive cross-validation, in the blue module, according to the MM, GS and K.in index, gene_8826, gene_7458, gene_61, gene_1741, and gene_11087 were the top network hub genes (Fig. 6A and Table S19). gene_7458 is the maintenance of mitochondrial morphology protein which is related to the phospholipid transport of mitochondrial outer membrane, participates in the assembly of mitochondrial outer membrane translocase complex, and transports mitochondrial amino acids to endoplasmic reticulum to synthesize laccase. Gene_ 1741 is a Laccase-3 gene, which is a key gene regulating laccase synthesis. Gene_61 belongs to the Sir2 family, which is a kind of NAD-dependent protein deacetylase. There was a lot of evidence in animals and plants, but in fungi, its function was studied in detail only in yeast. Studies have shown that yeast sir2 is related to senescence and oxidative stress. Sir2 is a key enzyme in the deacetylated gluconeogenesis pathway of hydrolyzing NAD + products, resulting in the loss of its catalytic activity [42-44], reducing intracellular sugar storage and delaying cell senescence. Besides, the hydrolysate of lignocellulose is considered to be a renewable raw material for microbial fermentation [45]. Among these hydrolysates, acetic acid has a very high concentration and has become a powerful inhibitor of microbial growth and fermentation. Sir2 enhanced the activity of acetyl-CoA synthetase and converted acetic acid to acetyl-CoA [46]. It is predicted that sir2 promotes the synthesis of acetyl-CoA, resulting in excessive reactive oxygen species (ROS) production, oxidative stress, and a series of effects on the regulation of cell cycle, apoptosis and autophagy $[47,48]$. On the other hand, low concentrations of reactive oxygen species $\left(\mathrm{H}_{2} \mathrm{O}_{2}\right.$ and $\left.\mathrm{OH}^{-}\right)$promoted the production of laccase by whiterot fungus, while high concentrations inhibited [49].

Interestingly, gene_11087 was a predictive transcription factor, and Pfam database comparison found that SNF2 family N-terminal domain (7.3e-61), Helicase conserved C-terminal domain (3.1e-18), Zinc finger(C3HC4) $(0.00015)$, but its protein function was not clear, through annotation can only be related to ATP binding (GO:0005524), zinc ion binding (GO:0008270). Snf2 family proteins can affect gene expression by using the energy hydrolyzed by ATP to change the interaction between histone and DNA, and most of the Snf2 proteins related to chromatin remodeling form complexes, which are likely to change the activity of core ATP enzymes [50,51]. But gene_11087 only has SNF2 family domain, whether it can influence ATP is unknown, and no research has shown whether it is related to the expression of laccase, which needs to be further explored. Therefore, the co-expression pattern of genes in the blue module and its regulatory factor (TF) is noteworthy. We predicted a total of 74 genes from 43 families (Table S12). At the same time, among the 66 highly linked genes, it was predicted that 4 transcription factors gene_9051,gene_9414,gene_9848,gene_9806 belonged to the AGC-PI, C2H2, CAMK_AMPK, IWS1 family (Fig. 6B and Table S12), which may regulate key circuits in the process of laccase secretion. In 
addition to the same hub gene in the turquoise module, gene_5464 was another special gene, mitochondrial inner membrane protein, succinate dehydrogenase cytochrome B small subunit (Table S19), which participated in the TCA cycle and the metabolic transfer activates redox metabolic systems, such as lignin, laccase, manganese peroxidase and cytochrome P450s [52]. Our research also has some limitations. First of all, there were many genes in the module. According to the index of GS, KIN, MM, we only selected the first 30 and 50 genes in the module, which may be biased in the study of laccase regulatory genes to some extent. Second, although the HUB genes in the two modules have been confirmed to be related to activity in different data sets and laccase expression as well as in some literature comments, a large number of experiments are still needed to verify these findings.

In our study, the genes located at the center of the network showed the strongest correlation with the laccase activity and DEGs. The key central genes of laccase activity were identified by WGCNA. In the citrate cycle (TCA cycle) (ko00020) signal pathway, the key HUB gene was gene_7458.In Nicotinate and nicotinamide metabolism (ko00760) signal pathway, the key HUB gene was gene_61. In the regulation of mitophagy-yeast (ko04139) signal pathway, the key HUB gene was gene_7458. The other were gene_1741(laccase3) and gene_11087 (SNF2 family unidentified protein). These central genes were consistent with DGEs. Therefore, we speculate that these key central genes are essential for laccase activity.

\section{Conclusion}

Transcriptome analysis of wood-treated L. gibbosa showed that transition metal ion transport, phenylpropanoid catabolic process, lignin catabolic process, lignin metabolic process, flavin adenine dinucleotide binding, antioxidant activity, oxidoreductase activity was enriched in the adjacent period, which was related to the degradation of lignin. There is an energy-related DEDS for Citrate cycle (TCA cycle), Glycolysis / Gluconeogenesis, and Carbon metabolism. laccase-1,2-oxoglutarate-Fe(II) type oxidoreductase, peroxisomal hydratase-dehydrogenase, Dual-functional monooxygenase, NADPHdependent 1-acyldihydroxyacetone phosphate reductase, dual-functional monooxygenase/methyltransferase psoF, Ferric reductase transmembrane component 1, Manganese peroxidase 3, P450 and ABC transporters were involved in lignin degradation by L. gibbosa at different stages. WGCNA combined with HUB gene network analysis revealed the key regulators of laccase activity.gene_7458,gene_61,gene_7458,gene_1741,gene_11087 and other pivotal genes were in the Citric acid cycle (TCA cycle), Nicotinate and nicotinamide metabolism (ko00760), Regulation of mitophagy yeast (ko04139). These genes were consistent with DGEs. To sum up, optimization of the fungal lignin degradation system is not only to strengthen the extracellular enzyme system but also a comprehensive metabolic structure design is needed. Therefore, we speculate that these key central genes are very important for laccase activity and deserve further attention. It is helpful to understand the production mechanism of $L$. gibbosa laccase and improve the activity of $L$. gibbosa laccase, which can be used to increase the rate of lignin degradation by L. gibbosa. 


\section{Methods}

\section{Sample mycelium}

L. gibbosa punched the bacterial cake with a $5 \mathrm{~mm}$ punch and transferred it to a conical flask (LNAS, Low Nitrogen Asparagine Succinic acid and $5 \mathrm{~mL} 15 \%$ glucose). Each conical flask was added 5 pieces of bacterial cakes. After cultured at $27^{\circ} \mathrm{C}$ for 10 days, it was recorded as CK group. Then, $2 \mathrm{~g}$ sawdust was added to each conical flask. On the 3rd, 5th, 7th, and 11th day, the extracted hyphae were recorded as $M X 1, M X 2, M X 3, M X 4$, and stored at- $80^{\circ} \mathrm{C}$ until RNA extraction. Each group consists of 3 samples as biological repetition, and each sample is also composed of 5 flasks.

The laccase activity was determined by an ultraviolet spectrophotometer at $420 \mathrm{~nm}$ to detect the oxidation rate of ABTS (2,2' -diazo (3-ethyl benzothiazolin-6-sulfonic acid). The reaction system was $1 \mathrm{~mL}$, of which $50 \mathrm{mmol} \cdot \mathrm{L}^{-1}$ sodium malonate buffer was $850 \mu \mathrm{L}, 20 \mathrm{mmol} \mathrm{L}-1$ ABTS solution was $50 \mu$ $L$, and the enzyme solution diluted was $100 \mu \mathrm{L}$. The increase of absorbance at $420 \mathrm{~nm}$ in the reaction 3 min was determined. And the enzyme amount required to convert 1 mol ABTS per minute was defined as laccase activity unit $\left(\left(\mathrm{UL}^{-1}\right)\right.$.

\section{RNA extraction, library construction, RNA sequencing and assembly}

From 15 samples, Trizol reagent (Invitgen, USA) was used to extract total RNA. Ultraviolet spectrophotometer and agarose gel electrophoresis were used to detect the quality of extracted RNA samples, and the Agilent 2100 bioanalyzer (Agilent Technologies, Palo Alto, CA, USA) was used to evaluate RNA integrity. The prepared cDNA libraries were sequenced on IlluminaHiSeqX-10 by BIOMARKER Biotechnology Co., Ltd. (Beijing, China). Import the Bcltofastq1.8.4 software from the original reading to carry on the base recognition work, after removing the joint, get the clean data, and Qscore < 30. Then use TopHat2 (version 2.0.3.12) to map the clean readings of each sample to the reference genome [53]. Based on the Cufflinks software, the FPKM method quantified the gene expression level by comparing the location information on the genome [54].

\section{Identification of differentially expressed genes (DEGs)}

The edgeR package (http://www.rproject.org/) was used to identify DEGs across groups with fold changes $\geq 2$ and a false discovery rate $(F D R)<0.05$. Then, DEGs were had to enrichment analysis of $G O$ (Gene Ontology) and KEGG (Kyoto Encyclopedia of Genes and Genomes) pathways by using the hypergeometric distribution orithm.

\section{Validation of gene expression by qRT-PCR}

To verify the effectiveness of high-throughput transcriptome sequencing, 4 genes from DEGs were selected for qRT-PCR analysis. Primer 6.0 software was used to design the primers, which were completed by Sangon Biotech (Shanghai, China)(Table S20).GPD gene was selected as the housekeeping gene 
[55].ABI Stepone plus was used for real-time PCR detection, and qPCR was performed following the protocol provided by the TaKaRa One-Step RT-PCR Kit (Baori medical technology (Beijing) co., LTD, Beijing, China ) Compared to using the threshold cycle $\left(2^{-\Delta \Delta C t}\right)$ method of gene expression.

\section{Construction of the weighted gene co-expression network}

There were 15 transcriptome samples (5-time points, 3 replicates each). Co-expression networks were constructed using the WGCNA (v1.69) package in R [20]. The normalized (screened for missing values) gene expression matrix was imported into WGCNA to construct co-expression modules. Gene expression matrix was searched for a suitable soft threshold for building gene networks using a scale-free topology model [56]. After a threshold filter, the final selection is $\beta=22$. To evaluate the correlation between gene expression, the adjacency matrix was transformed into Topological Overlap Matrix (TOM) [57], and the Dissimilar Topological Matrix (dissTOM = 1-TOM) was used to carry out matrix clustering and module partitioning by the dynamic shearing an orithm. The minimum number of elements in a module is 30 (minModule Size $=30$ ), and the threshold for the merging of a similar module is 0.2 (CutHeight $=0.2$ ). Then, to identify biologically significant modules, module eigengenes (MEs) were used to calculate the correlation coefficients. MEs were considered to be an overview of gene expression in the module and are defined as the representation of the basic components of the given module. The Pearson correlation coefficient in the corPvalueStudent () function was used to calculate the correlation between laccase matrix and module feature gene matrix to obtain the $p$-value. The smaller the $p$-value, the two matrixes were the more significant the correlation. In order to screen laccase related specific modules, the modules of $|r|>0.60$ and $p$-value $<0.001$ were selected as the specific modules for further analysis. The networks were visualized using Cytoscape_3.7.1.

\section{Identification of significant hub genes}

Hub genes are generally characterized by high GS, high MM, and high K.in. In a module, it is highly connected with other genes, so it has high functional significance and is often located in the center of the module network [24]. The biological functions of each module were analyzed by enrichment analysis of GO and KEGG pathways. And, the protein sequence of each module was submitted to Plant TFDB (http://planttfdb.cbi.pku.edu.cn/prediction.php) analysis and forecasting, whereby obtained transcription factor [58].

\section{Declarations}

Ethics approval and consent to participate: Not applicable.

Consent for publication: The authors declared that they agree to publish this work.

\section{Availability of data and materials:}

All analyses tools used in the study are publicly available. The reference genome of this species is Tragib1 in JGI (http://genome.jgi.doe.gov/Tragib1/Tragib1.home.html). The edgeR and WGCNA package are from R (http://www.rproject.org/). 
All data generated or analysed during this study are included in this published article and its supplementary information files.

Competing interest: The authors declared that they have no conflicts of interest to this work.

Funding: This work was supported by grants from National natural science foundation of China. The funders had key role in the data analysis and interpretation of data.

Authors' contributions $\square$ J. C. and Y. C. designed the work; Q. Z. and X.H. performed the experiments; J. C. analyzed the data and wrote the paper.

\section{Acknowledgements:}

We are grateful to the Sangon Biotech (Shanghai, China) and BIOMARKER Biotechnology Co., Ltd. (Beijing, China) for technical and equipment assistance.

\section{References}

1. Asgher M, Bhatti HN, Ashraf M, Legge RL. Recent developments in biodegradation of industrial pollutants by white rot fungi and their enzyme system. BIODEGRADATION. 2008;19(6):771-83.

2. Pollegioni L, Tonin F, Rosini E. Lignin degrading enzymes. FEBS J. 2015;282(7):1190-213.

3. Sharma P, Goel R, Capalash N. Bacterial laccases. WORLD J MICROB BIOT. 2007;23(6):823-32.

4. Lu S, Li Q, Wei H, Chang MJ, Chiang VL. Ptr-miR397a is a negative regulator of laccase genes affecting lignin content in Populus trichocarpa. Proc Natl Acad Sci USA. 2013;110(26):10848-53.

5. Dittmer NT, Suderman RJ, Jiang H, Zhu YC, Gorman MJ, Kramer KJ, Kanost MR. Characterization of cDNAs encoding putative laccase-like multicopper oxidases and developmental expression in the tobacco hornworm, Manduca sexta, and the malaria mosquito, Anopheles gambiae. Insect Biochemistry Molecular Biology. 2004;34(1):29-41.

6. Baldrian P. Fungal laccases-occurrence and properties. FEMS MICROBIOL REV. 2006: 30 (2): 215242.

7. Yan, Xu, Bian, Li Z. Expression Profile of Laccase Gene Family in White-Rot Basidiomycete Lentinula edodes under Different Environmental Stresses. GENES-BASEL. 2019;10(12):1045.

8. Giardina P, Faraco V, Pezzella C, Piscitelli A, Vanhulle S, Sannia G. Laccases: a never-ending story. Cellular Molecular Life Sciences. 2010;67(3):369-85.

9. Alessandra P. Cinzia, Pezzella, Paola, Giardina, Vincenza, Faraco, Giovanni, Sannia, Heterologous laccase production and its role in industrial applications. Bioengineered Bugs. 2014;1:254-64.

10. Bettin F, Cousseau F, Martins K, Boff NA, Zaccaria S, Moura Da Silveira M, Dillon P. A.J., Phenol removal by laccases and other phenol oxidases of Pleurotus sajor-caju PS-2001 in submerged cultivations and aqueous mixtures. J ENVIRON MANAGE. 2019;236:581-90. 
11. Xiaojian M, Lu L, Qingqing L, Yunyun L, Li Y, Lixin M, Chao Z. High-level expression of a bacterial laccase, CueO from Escherichia coli K12 in Pichia pastoris GS115 and its application on the decolorization of synthetic dyes. ENZYME MICROB TECH. 2017; (103): 34-41.

12. Linares NC, Fernández F, Loske AM, Gómez-Lim MA. Enhanced Delignification of Lignocellulosic Biomass by Recombinant Fungus Phanerochaete chrysosporium Overexpressing Laccases and Peroxidases. Journal of Molecular Microbiology Biotechnology. 2018;28(1):1.

13. Liu W, Chao Y, Liu S, Bao H, Qian S. Molecular cloning and characterization of a laccase gene from the basidiomycete Fome lignosus and expression in Pichia pastoris. Applied Microbiology Biotechnology. 2003;63(2):174-81.

14. Robertson PL, Maas M, Goldblatt J. Molecular analysis of a laccase gene from the white rot fungus Pycnoporus cinnabarinus. Applied Environmental Microbiology. 1998;64(5):1766-72.

15. Xiao YZ, Hong YZ, Li JF, Hang J, Tong PG, Fang W, Zhou CZ. Cloning of novel laccase isozyme genes from Trametes sp. AH28-2 and analyses of their differential expression. Applied Microbiology Biotechnology. 2006;71(4):493-501.

16. Zhao Q, Chi Y, Zhang J, Feng L. Transcriptome Construction and Related Gene Expression Analysis of Lenzites gibbosa in Woody Environment. Scientia Silvae Sinicae. 2019;55(8):95-105.

17. Yan H Molecular cloning of cDNA gene encoding a manganese peroxidase from Pseudotrametes gibbosa and heterologous expression of this gene in Pichia pastoris. Doctoral Thesis, Northeast Forestry University, China, 2009.

18. Miaoiao Z. Molecular cloning of gene encoding a Laccase from Lenzites gibbosa and heterologous expression of this gene in Pichiapastoris. Doctoral Thesis, Northeast Forestry University, China, 2012.

19. Wu S Cloning and expression analysis of three manfanese perxidase genes from Lenzites gibbosa. Master Thesis, Northeast Forestry University, China, 2012.

20. LangfelderSteve P. Horvath, WGCNA: an R package for weighted correlation network analysis. BMC BIOINFORMATICS. 2008;9:559.

21. He, Li, Xiaojiao, Han, Wenmin Q, Dong, Xu Y. Wang, Identification and expression analysis of the GDSL esterase/lipase family genes, and the characterization of SaGLIP8 in Sedum alfredii Hance under cadmium stress. PEERJ. 2019.

22. Hong Z, Ying, Fu, Huan, Guo, Lu C, Wang. Weining, Transcriptome and Proteome-Based Network Analysis Reveals a Model of Gene Activation in Wheat Resistance to Stripe Rust. INT J MOL SCI. 2019.

23. Zhang Y, Yao J, Hu F, Jiang J, Fan X, Jia Y, Ran W, Liu C. Identification of the defense-related gene VdWRKY53 from the wild grapevine Vitis davidii using RNA sequencing and ectopic expression analysis in Arabidopsis. HEREDITAS. 2019;156(1):14.

24. Lou Y, Tian GY, Song Y, Liu YL, Yang J. Characterization of transcriptional modules related to fibrosing-NAFLD progression. SCI REP-UK. 2017;7(1):4748.

25. Vrsanska M, Voberkova S, Langer V, Palovcikova D, Moulick A, Adam V, Kopel P. Induction of Laccase, Lignin Peroxidase and Manganese Peroxidase Activities in White-Rot Fungi Using Copper 
Complexes. MOLECULES. 2016;21(11):1553.

26. Baoyan H, Hua Y, Hui P, Jinshao Y, Yan L, Sufeng P. Study on the effect of metal ions towards biodegradation pathway of decabrominated diphenyl ether by white rot fungus. Journal of Safety Environment. 2012;12(6):85-9.

27. Omura T. Forty Years of Cytochrome P450. Biochemical \&. Biophysical Research Communications. 1999;266(3):690-8.

28. Sun J, Zhang T, Li Y, Wang X, Chen J. Functional characterization of the ABC transporter TaPdr2 in the tolerance of biocontrol the fungus Trichoderma atroviride T23 to dichlorvos stress. BIOL CONTROL. 2018; 129.

29. Ferreira $P$, Carro J, Serrano A, Martínez AT. A survey of genes encoding $\mathrm{H}_{2} \mathrm{O}_{2}$-producing GMC oxidoreductases in 10 Polyporales genomes. MYCOLOGIA. 2015;107(6):1105-19.

30. Kracher D, Scheiblbrandner S, Felice AKG, BresImayr E, Preims M, Ludwicka K, Haltrich D, Eijsink VGH, Ludwig R. Extracellular electron transfer systems fuel cellulose oxidative degradation. SCIENCE. 2016;352(6289):1098-101.

31. Fumiko M. Motoyuki, Shimizu, Hiroyuki, Wariishi, Proteomic and Metabolomic Analyses of the WhiteRot Fungus Phanerochaete chrysosporium Exposed to Exogenous Benzoic Acid. J PROTEOME RES. 2008;7(6):2342 2350.

32. Curnow AW, Tumbula DL, Pelaschier JT, Min B, Söll D. Glutamyl-tRNAGIn amidotransferase in Deinococcus radiodurans may be confined to asparagine biosynthesis. P NATL ACAD SCI USA. 1998;95(22):12838-43.

33. Dickman MB, Yarden O. Serine/Threonine Protein Kinases and Phosphatases in Filamentious Fungi. Fungal Genetics Biology. 1999;26(2):99-117.

34. Chen F, Chen X, Su X, Qin L, Huang Z, Tao Y, Dong Z. An Ime2-like mitogen-activated protein kinase is involved in cellulase expression in the filamentous fungus Trichoderma reesei. BIOTECHNOL LETT. 2015; (37): 2055-2062.

35. Shanshan W, Liangcai L, ShiRu J, Chaoguang T. Cellulase expression analysis of serine/threonine kinase gene deletion mutants of Neurospora crassa. Microbiology China. 2017;44(06):1303-11.

36. Xinxing Lv, Weixin, Zhang, Guanjun, Chen. Weifeng, Liu, Trichoderma reesei Sch9 and Yak1 regulate vegetative growth, conidiation, and stress response and induced cellulase production. J MICROBIOL. 2015.

37. Wang R, Zeng G, Yu H, Huang G. Lignin Degradation Mechanism by Microbes. J MICROBIOL. 2008;28(3):59-63.

38. Miyauchi S, Rancon A, Drula E, Hage H, Chaduli D, Favel A, Grisel S. Integrative visual omics of the white-rot fungus Polyporus brumalis exposes the biotechnological potential of its oxidative enzymes for delignifying raw plant biomass. BIOTECHNOL BIOFUELS. 2018;11:201.

39. Ning D, Wang H, Involvement of Cytochrome P450 in Pentachlorophenol Transformation in a White Rot Fungus Phanerochaete chrysosporium. PLOS ONE. 2012; 7. 
40. Khajamohiddin S, Karabo S, Sekhar PN, Jack T. Systematic identification and evolutionary analysis of catalytically versatile cytochrome p450 monooxygenase families enriched in model basidiomycete fungi. PLOS ONE. 2014;9(1):e86683.

41. Jacqueline MacDonald, Hitoshi, SuzukiEmma R, Master. Expression and regulation of genes encoding lignocellulose-degrading activity in the genus Phanerochaete. Applied Microbiology Biotechnology. 2012;94(2):339 351.

42. Lin YY, Lu JY, Zhang J, Walter W, Dang W, Wan J, Tao SC, Qian J, Zhao Y, Boeke JD. Protein Acetylation Microarray Reveals that NuA4 Controls Key Metabolic Target Regulating Gluconeogenesis. CELL. 2009;136(6):1073-84.

43. Orlandi I, Stamerra G, Strippoli M, Vai M. During yeast chronological aging resveratrol supplementation results in a short-lived phenotype Sir2-dependent. Redox Biology. 2017;12:745-54.

44. Wang L, Jin W, Chen Y, Wang J. Fundion and Mechanism of Sir2 Gene Family. Chinese Joumal of Cell Biology. 2006; (28): 822-826.

45. Almario MP, Reyes LH, Kao KC. Evolutionary Engineering of Saccharomyces cerevisiae for Enhanced Tolerance to Hydrolysates of Lignocellulosic Biomass. Biotechnology Bioengineering. 2013;110(10):2616-23.

46. Vall-Ilaura N, Mir N, Garrido L, Vived C, Cabiscol E. Redox control of yeast Sir2 activity is involved in acetic acid resistance and longevity. REDOX BIOL. 2019;24:101229.

47. Ding Y, Zhu W, Sun R, Yuan G, Sun J. Diphenylene iodonium interferes with cell cycle progression and induces apoptosis by modulating $\mathrm{NAD}(\mathrm{P}) \mathrm{H}$ oxidase/ROS/cell cycle regulatory pathways in Burkitt's lymphoma cells. ONCOL REP. 2015;33(3):1434-42.

48. Rada B, Leto TL. Oxidative Innate Immune Defenses by Nox/Duox Family NADPH Oxidases. Contrib Microbiol. 2008;15(15):164-87.

49. Tian Q, Wang X, Zhang Y, Zhengbing G, Cai Y, Liao X. Effect of Reactive Oxygen Species on Laccase Synthesis of White-Rot Fungus. Journal of Food Science Biotechnology. 2019;38(5):103-10.

50. Cairns BR. Chromatin remodeling complexes: strength in diversity, precision through specialization. CURR OPIN GENET DEV. 2005;15(2):185-90.

51. Hargreaves DC, Crabtree GR. ATP-dependent chromatin remodeling: genetics, genomics and mechanisms. CELL RES. 2011;21(3):396-420.

52. Fumiko M. Motoyuki, Shimizu, Hiroyuki, Wariishi, Proteomic and Metabolomic Analyses of the WhiteRot Fungus Phanerochaete chrysosporium Exposed to Exogenous Benzoic Acid. J PROTEOME RES. 2008;7(6):2342-50.

53. Kim D, Pertea G, Trapnell C, Pimentel H, Kelley R. TopHat2: accurate alignment of transcriptomes in the presence of insertions, deletions and gene fusions. GENOME BIOL. 2013;14(4):R36.

54. Florea L, Song L, Salzberg SL. Thousands of exon skipping events differentiate among splicing patterns in sixteen human tissues. F1000res. 2013;2:188. 
55. Ullmannová V, Haskovec C. The use of housekeeping genes (HKG) as an internal control for the detection of gene expression by quantitative real-time RT-PCR. Folia Biol. 2003;49(6):211-6.

56. Wang N, Wang R, Wang R, Chen S. Transcriptomics analysis revealing candidate networks and genes for the body size sexual dimorphism of Chinese tongue sole (Cynoglossus semilaevis). FUNCT INTEGR GENOMIC. 2018;18(3):327-39.

57. Zhang B, Horvath S. A general framework for weighted gene co-expression network analysis. Statistical Applications in Genetics Molecular Biology. 2005;4(1):Article17.

58. Jin J, Tian F, Yang DC, Meng YQ, Kong L, Luo J, Gao G. PlantTFDB 4.0: toward a central hub for transcription factors and regulatory interactions in plants. NUCLEIC ACIDS RES. 2017;45(D1):D1040-5.

\section{Supplementary Information}

Additional file 1: Supplementary Fig. 1. Quantitative real-time polymerase chain reaction (qRT-PCR) of DEGs.

Additional file 2: Supplementary Fig. 2. Clustering of module eigengenes.

Additional file 3: Supplementary Fig. 3. Modules Significance Correlation.

Additional file 4: Supplementary Fig. 4. Interaction of co-expression patterns for genes in the blue module.

Additional file 5: Supplementary Table 1. Transcriptome sequencing's data statistics.

Additional file 6: Supplementary Table 2. Comparisons of numbers of DEGs at different times.

Additional file 7: Supplementary Table 3. KEGG pathways enrichment in ten compared groups.

Additional file 8: Supplementary Table 4. GO enrichment of DEGs in different time periods.

Additional file 9: Supplementary Table 5. Five hub genes in blue module.

Additional file 10: Supplementary Table 6. Edge weight for blue module.

Additional file 11: Supplementary Table 7. Seven hub genes in turquoise module.

Additional file 12: Supplementary Table 8. Edge weight for module turquoise.

Additional file 13: Supplementary Table 9. GO enrichment of hub genes in blue module.

Additional file 14: Supplementary Table 10. KEGG pathway enrichment of hub genes in blue module.

Additional file 15: Supplementary Table 11. KEGG class of hub genes in blue module.

Additional file 16: Supplementary Table 12. Prediction of transcription factors in the blue module. 
Additional file 17: Supplementary Table 13. G0 enrichment of hub genes in turquoise module.

Additional file 18: Supplementary Table 14. KEGG pathway enrichment of hub genes in turquoise module.

Additional file 19: Supplementary Table 15. KEGG class of hub genes in turquoise module.

Additional file 20: Supplementary Table 16. Prediction of transcription factors in the turquoise module.

Additional file 21: Supplementary Table 17. The DEGs annotation in CKvsMX1.

Additional file 22: Supplementary Table 18. Gene expression changes of Secondary Metabolites in DEGs at different time period.

Additional file 23: Supplementary Table 19. Interesting genes annotation in two modules.

Additional file 24: Supplementary Table 20. Primers used in present study.

\section{Figures}

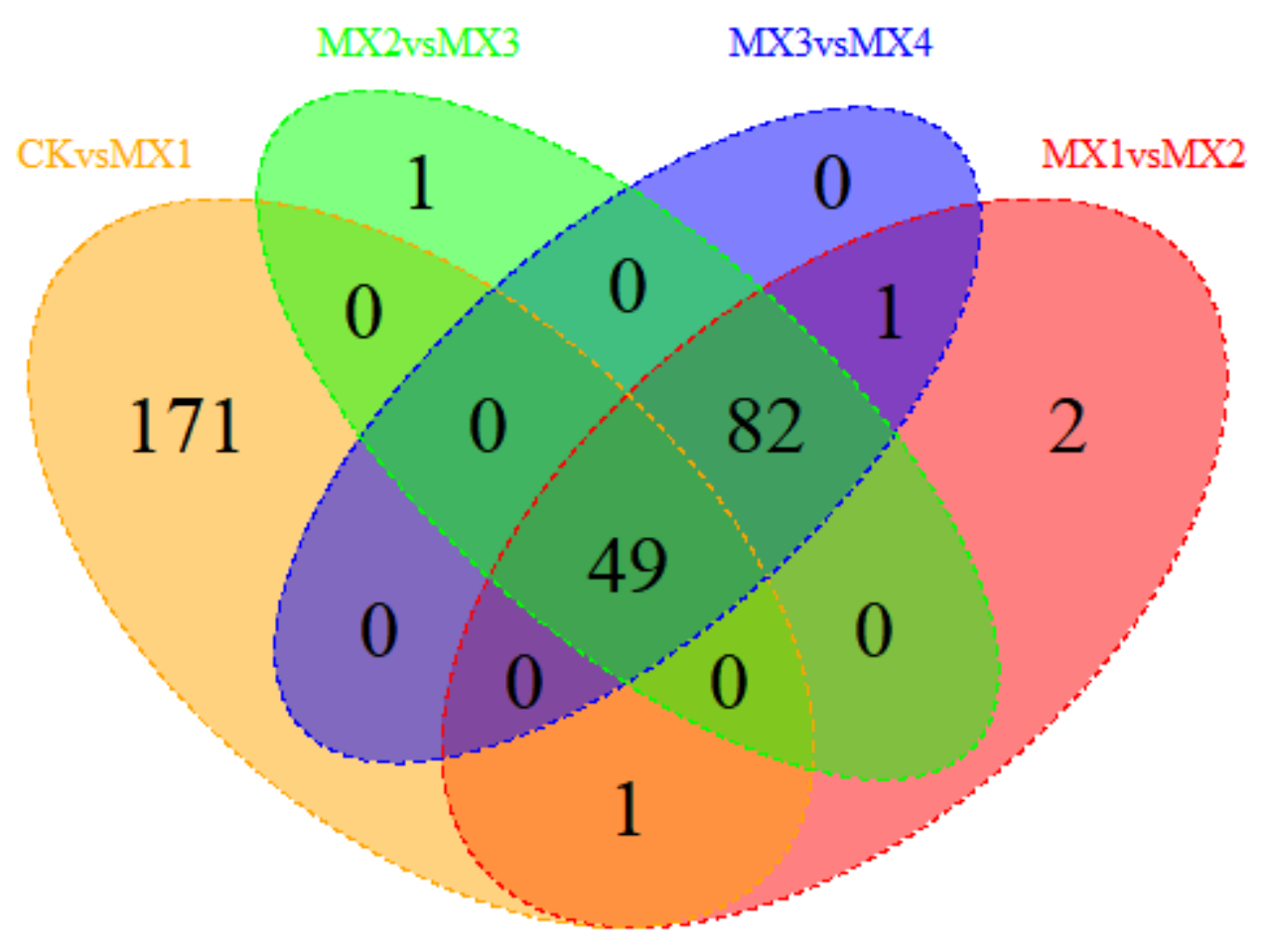

\section{Figure 1}

Venn diagram of GO enrichment in adjacent time point DEGs $(p<0.05)$ 


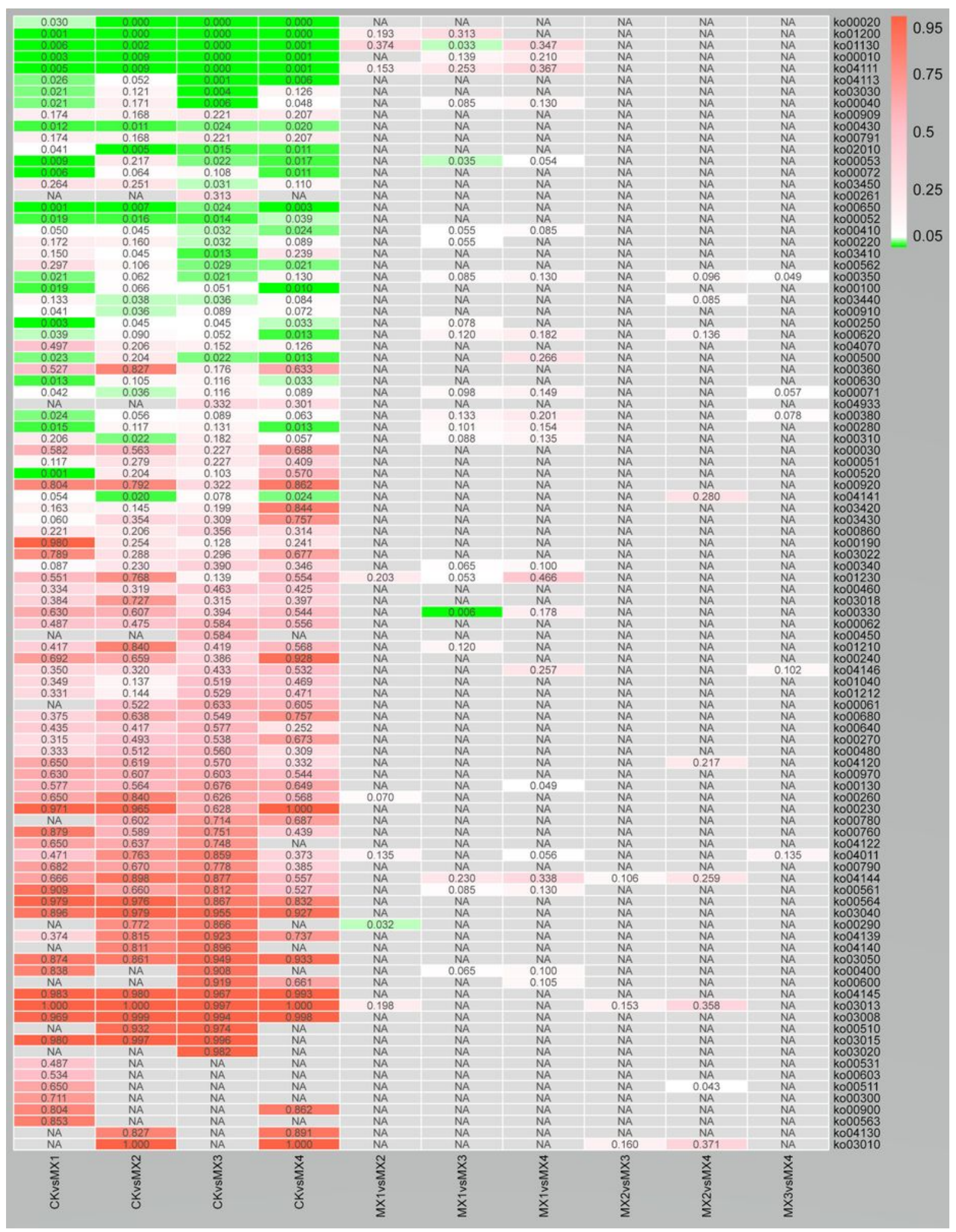

Figure 2

Heatmap of pathway enrichment analysis in DEGs 

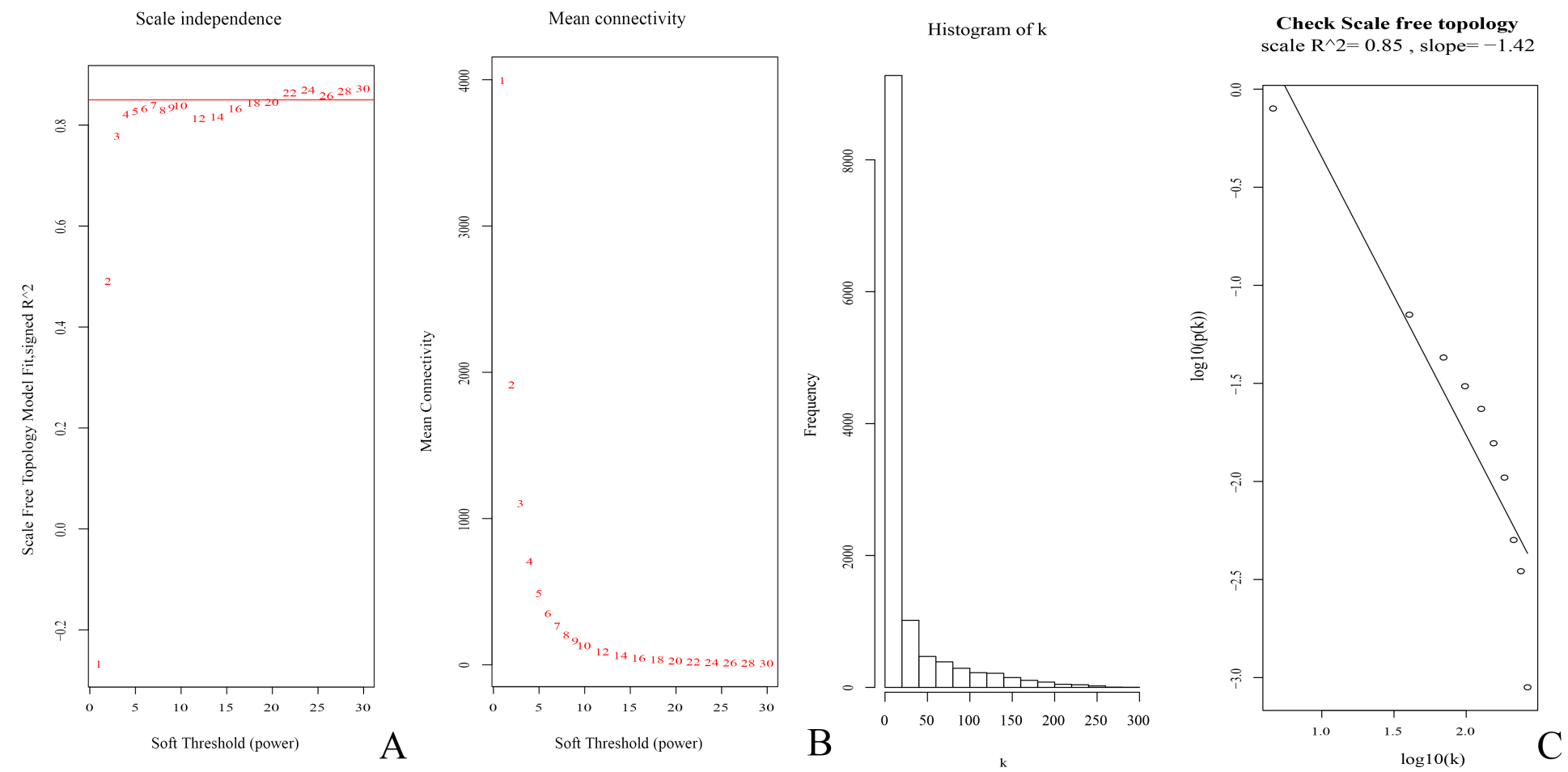

Figure 3

Selection of the soft-thresholding powers. A The panel showed the scale-free fit index versus softthresholding power; B The right panel displayed the mean connectivity versus soft-thresholding power. C Verifies the memory network with selected values R2 $=0.85$. 


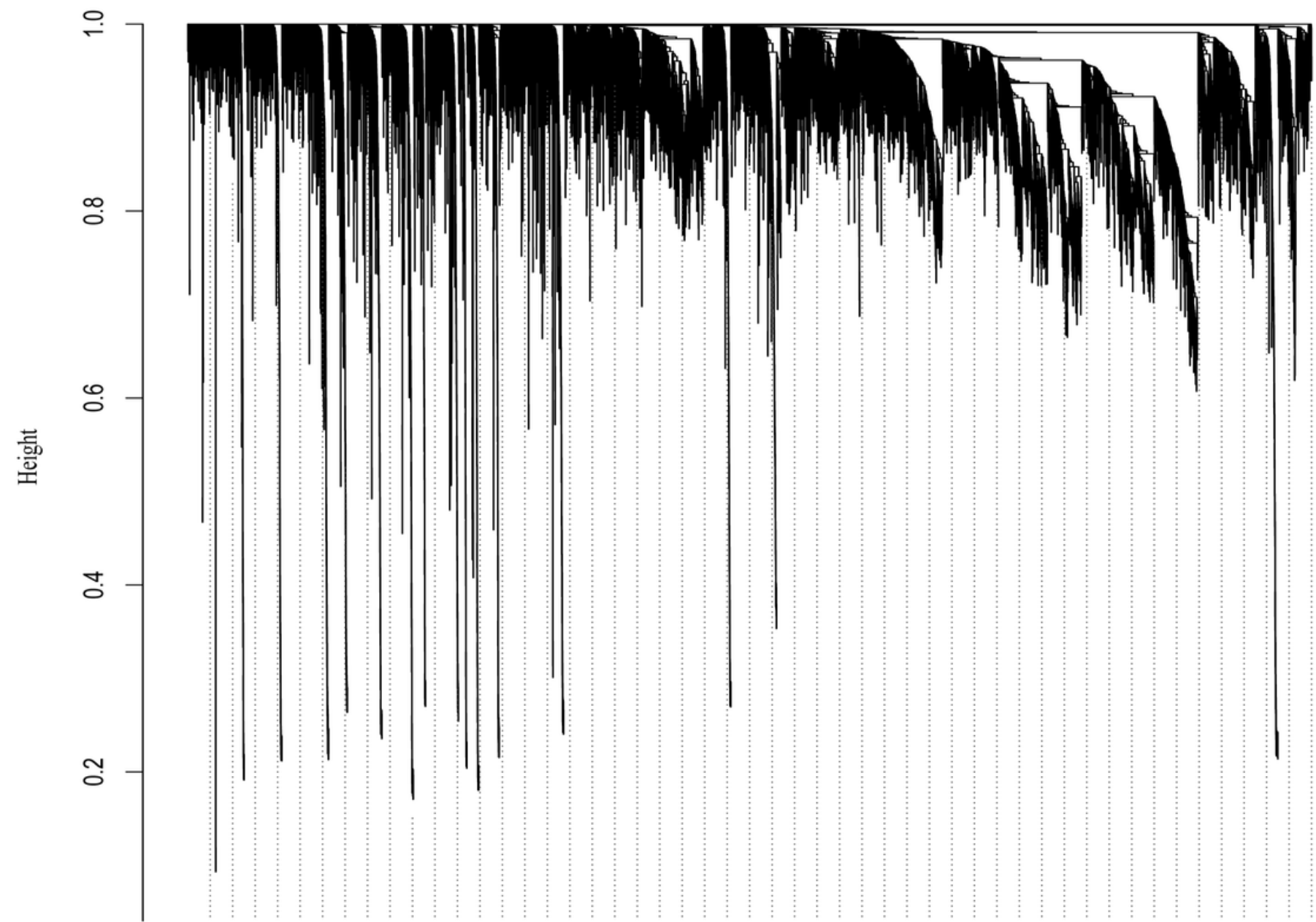

A,Dynamic Tree Cut

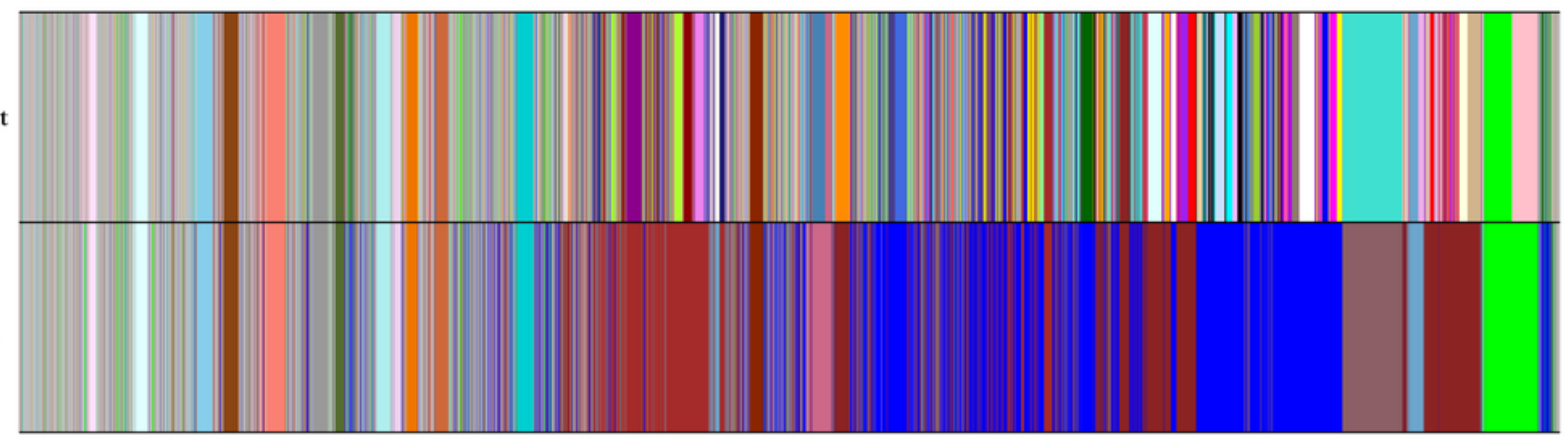

B, Merged dynamic

\section{Figure 4}

Clustering dendrograms of genes and module detecting A. Module division by dynamic tree cut, different colors represent different modules; B. Module division after merging similar modules 

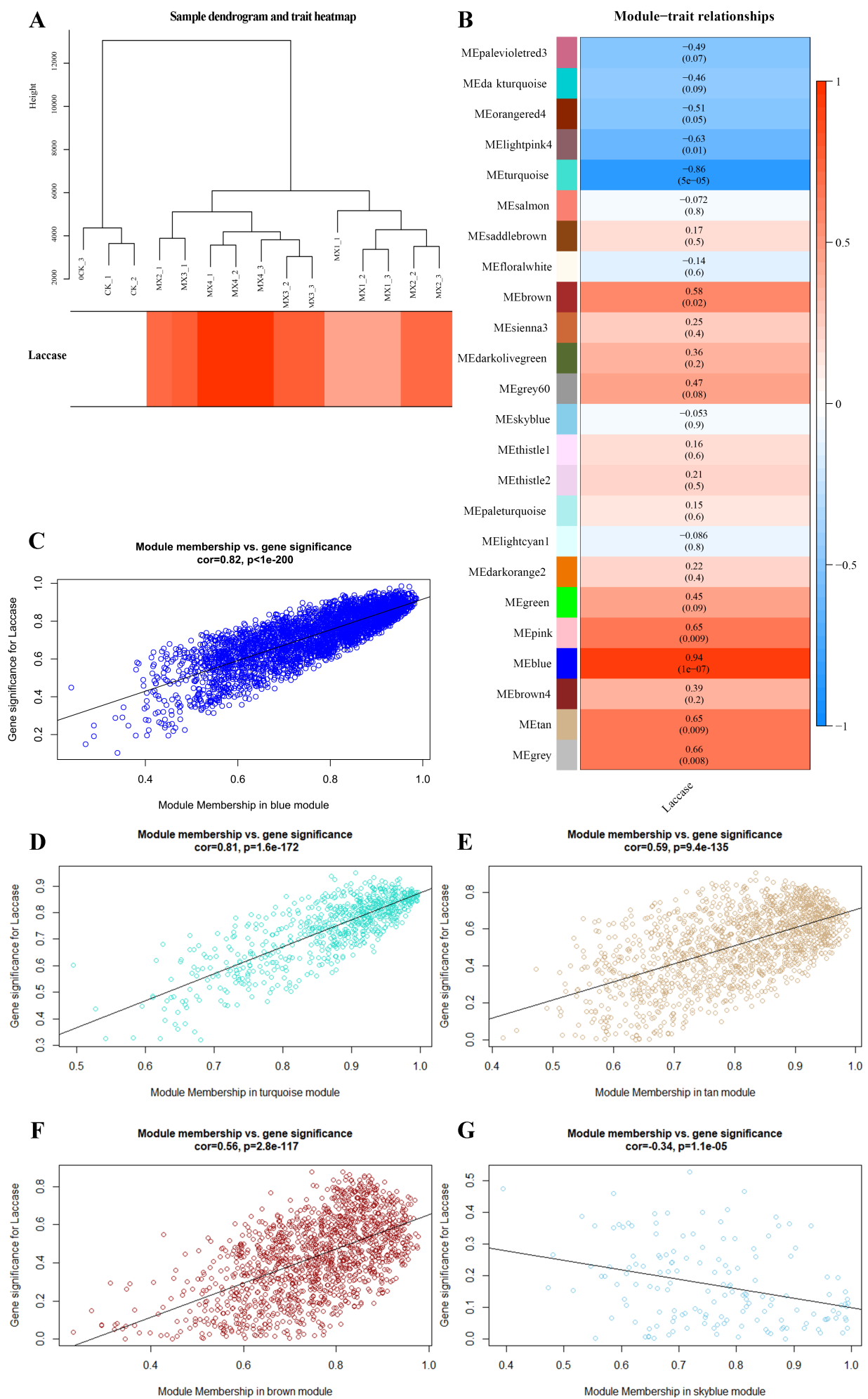

Figure 5

Correlation diagram of laccase activity. A. Sample dendrogram and trait heatmap; B. Module-trait; C. Module membership vs. gene significance in the blue module; D. Module membership vs. gene significance in the turquoise module; E. Module membership vs. gene significance in the tan module (Each dot represents a single gene in each module, drawn by GS on the y-axis and MM on the x-axis. Each graph shows the regression line, related values, and $p$ values.). 

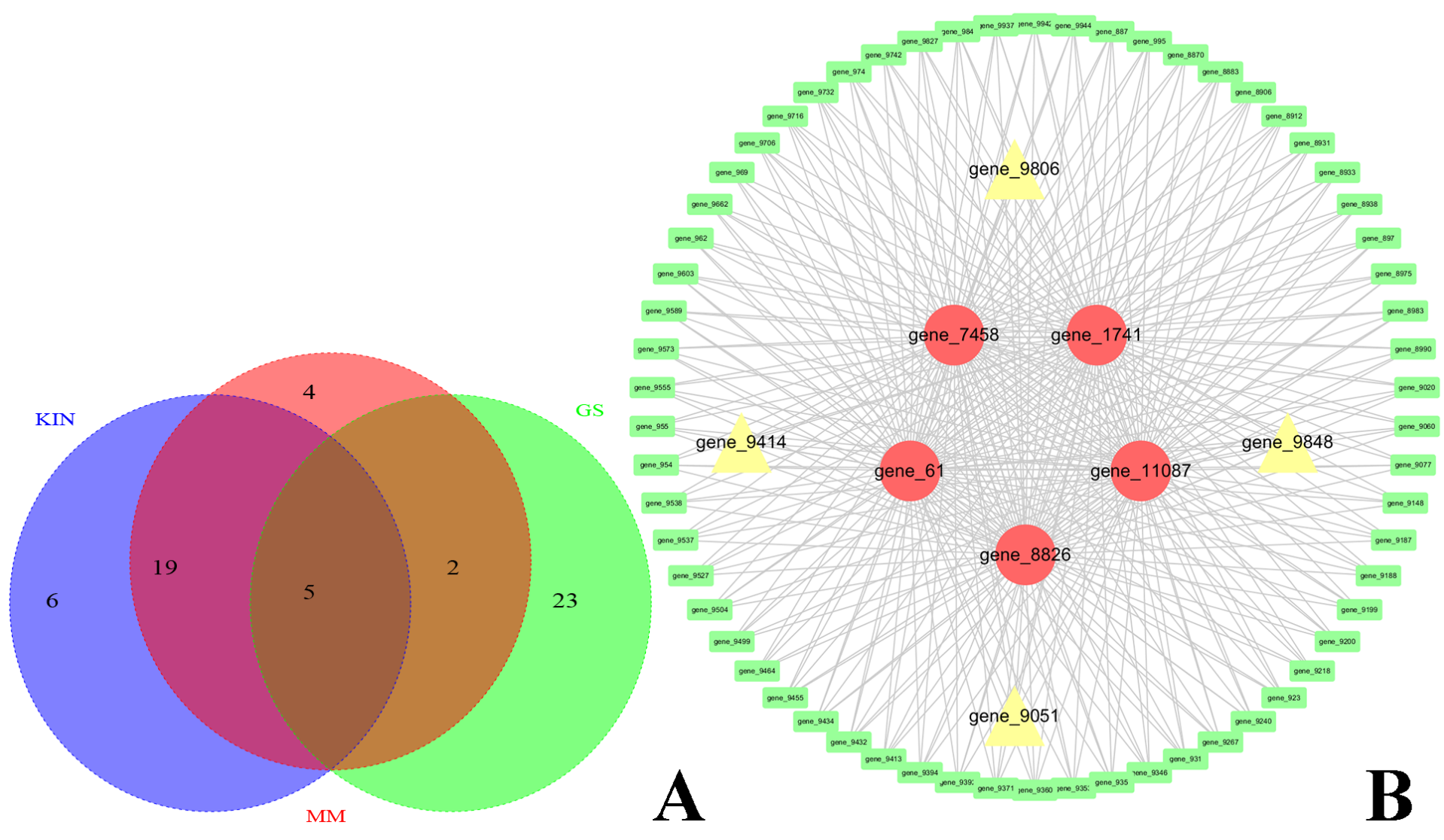

\section{Figure 6}

Characterization of the blue module. A. Venn diagram of blue module genes in the top of 30 based on high gene significance (GS), high module membership (MM), and high intramodular connectivity (K.in); B. Interaction of gene co-expression patterns of 66 highly relevant genes in the blue module, those were used for the construction of the network in Cytoscape 3.7.1. Note: The red markers indicate the key hub genes and the yellow markers indicate the transcription factors. 

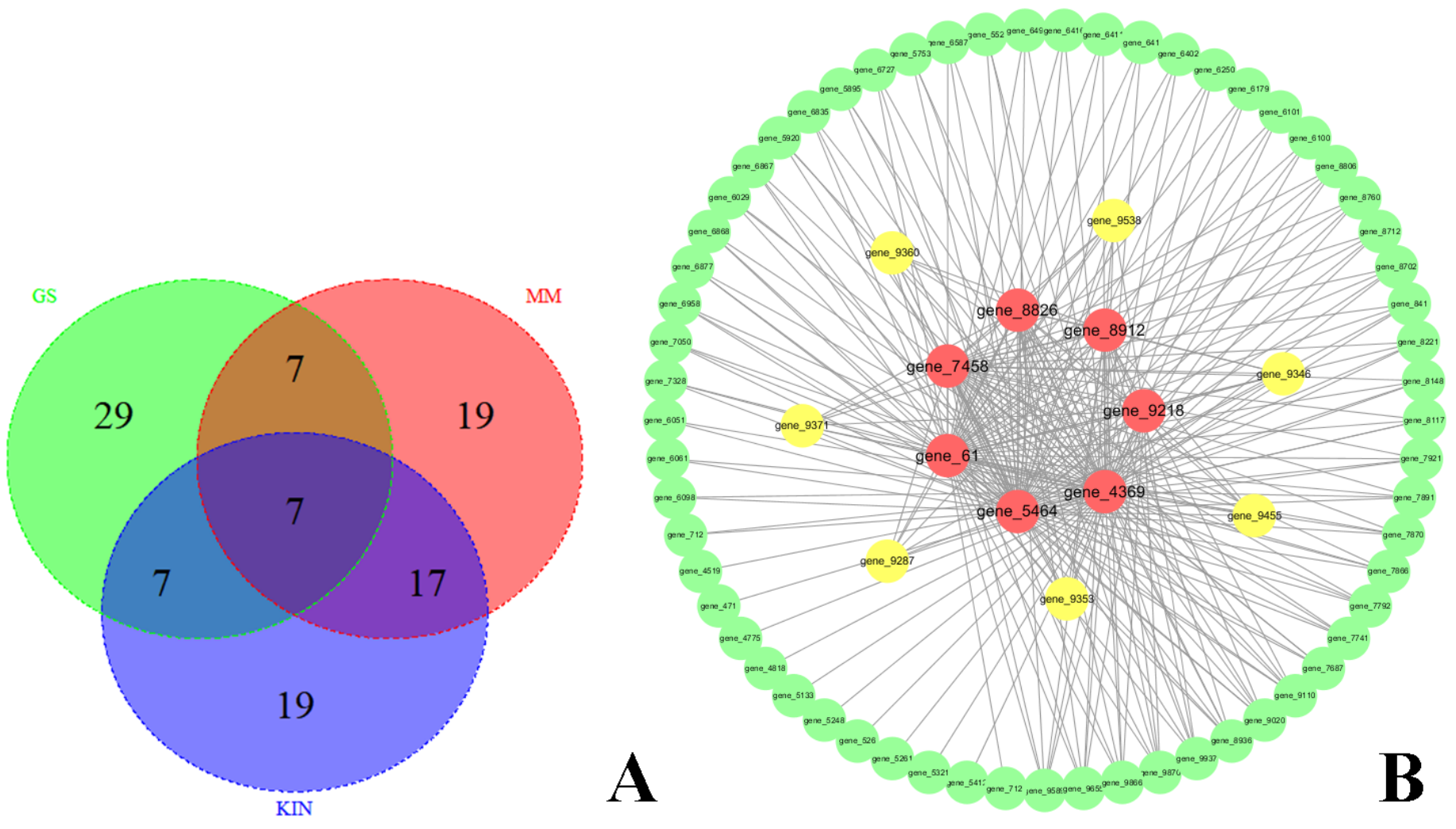

Figure 7

Characterization of the turquoise module. A. Venn diagram of turquoise module genes in the top of 50 based on high gene significance (GS), high module membership (MM), and high intramodular connectivity (K.in); B. Interaction of gene co-expression patterns in the turquoise module. 75 genes with the highest weight are in turquoise; those were used for the construction of the network in Cytoscape 3.7.1. Note: The red markers indicate the key hub genes and the yellow markers indicate the high correlation genes. 

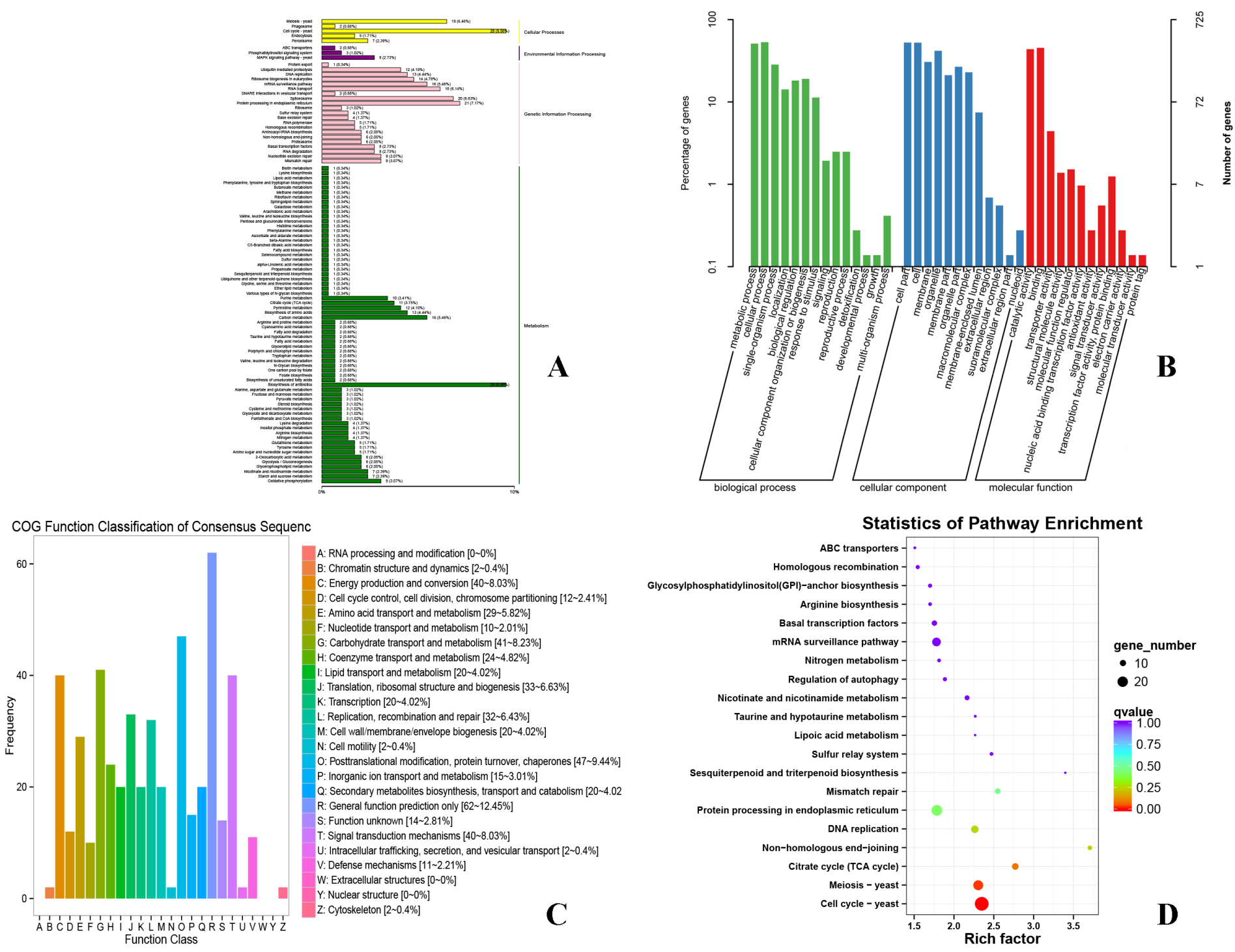

Figure 8

Feature analysis of blue module. A. Module hub genes KEGG classification; B. Module hub genes GO annotation; C. Module hub genes COG annotation; D. Module hub genes KEGG enrichment 

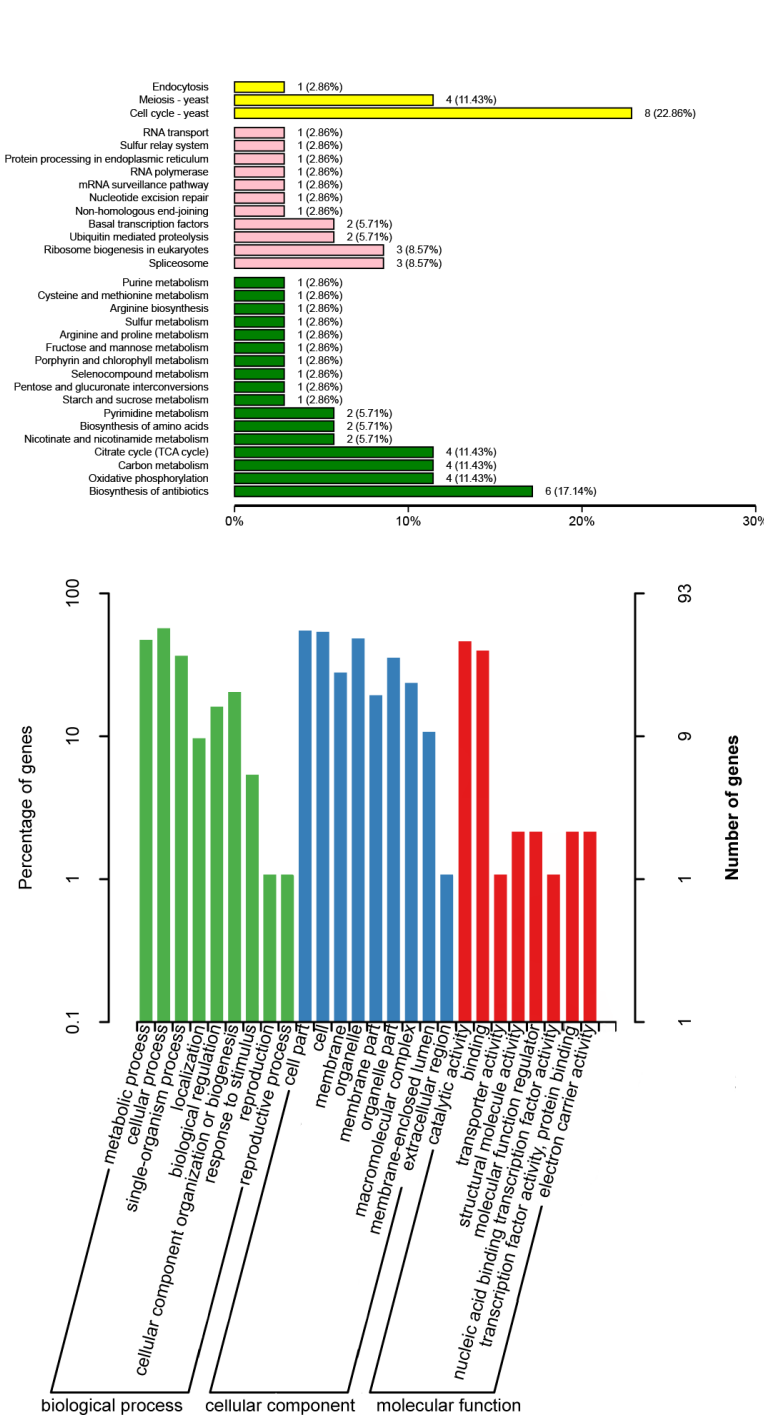

COG Function Classification of Consensus Sequenc

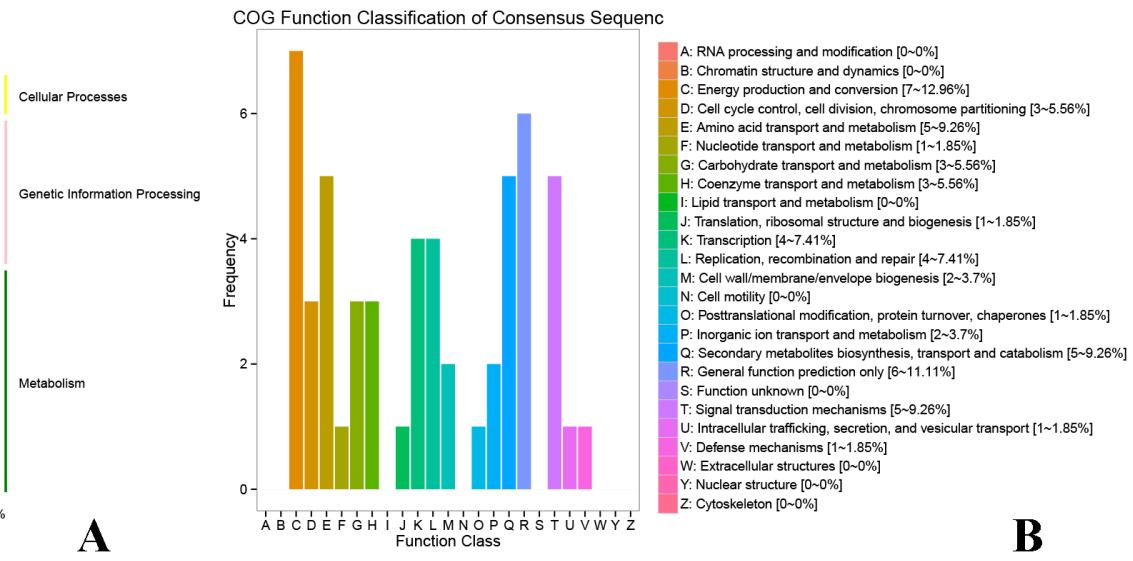

Statistics of Pathway Enrichment

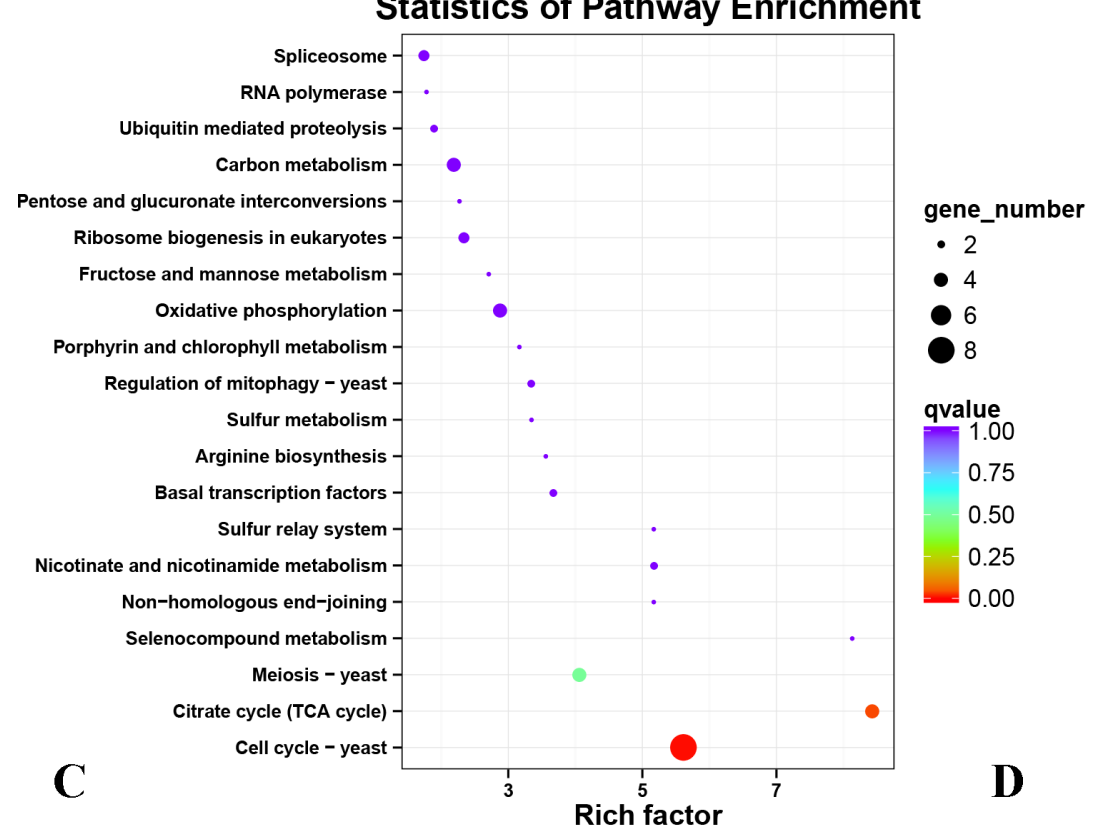

Figure 9

Feature analysis of the turquoise module. A. Module hub genes KEGG classification; B. Module hub genes COG annotation; C. Module hub genes GO annotation; D. Module hub genes KEGG enrichment

\section{Supplementary Files}

This is a list of supplementary files associated with this preprint. Click to download.

- TableS2..xIsx

- Tables3..xlsx

- Tables4..xlsx

- TableS5..xlsx

- TableS6..xIsx

- TableS7.Sevenhubgenesinturquoisemodule.xlsx 
- TableS8.Edgeweightformoduleturquoise.xIsx

- Tables9.GOenrichmentofhubgenesinbluemodule.xlsx

- TableS10..xlsx

- TableS11.KEGGclassofhubgenesinbluemodule.xlsx

- TableS12..xlsx

- TableS13.GOenrichmentofhubgenesinturquoisemodule.xlsx

- TableS14..xlsx

- TableS15.KEGGclassofhubgenesinturquoisemodule.xlsx

- TableS16..xlsx

- TableS17.DEGsannotationinCKvsMX1.xlsx

- TableS18..xlsx

- TableS19.Interestinggenesannotationintwomodules.xlsx

- TableS20.Primersusedinpresentstudy..xlsx

- Tables1..xlsx

- Figs4..pdf

- FigureS1.png

- FigS2.png

- FigS3ModulesSignificanceCorrelation.png 